\title{
Multiple Path Prediction for Traffic Scenes Using LSTMs and Mixture Density Models
}

\author{
Jaime B. Fernandez $\mathbb{1}^{\mathrm{a}}$, Suzanne Little $\mathbb{( \mathbb { b }}^{\mathrm{b}}$ and Noel E. O'Connor $\mathbb{D}^{\mathrm{c}}$ \\ Insight Centre for Data Analytics, Dublin City University, Dublin, Ireland \\ jaime.fernandezroblero5@mail.dcu.ie,suzanne.little@dcu.ie,noel.oconnor@dcu.ie
}

Keywords: $\quad$ Multiple Path Prediction, Traffic Scenes, LSTMs, MDNs, Time Series.

Abstract: $\quad$ This work presents an analysis of predicting multiple future paths of moving objects in traffic scenes by leveraging Long Short-Term Memory architectures (LSTMs) and Mixture Density Networks (MDNs) in a single-shot manner. Path prediction allows estimating the future positions of objects. This is useful in important applications such as security monitoring systems, Autonomous Driver Assistance Systems and assistive technologies. Normal approaches use observed positions (tracklets) of objects in video frames to predict their future paths as a sequence of position values. This can be treated as a time series. LSTMs have achieved good performance when dealing with time series. However, LSTMs have the limitation of only predicting a single path per tracklet. Path prediction is not a deterministic task and requires predicting with a level of uncertainty. Predicting multiple paths instead of a single one is therefore a more realistic manner of approaching this task. In this work, predicting a set of future paths with associated uncertainty was archived by combining LSTMs and MDNs. The evaluation was made on the KITTI and the CityFlow datasets on three type of objects, four prediction horizons and two different points of view (image coordinates and birds-eye view).

\section{INTRODUCTION}

Given a scene, knowing where an object is currently located is useful information to be able to interact in such environment. However, nowadays, knowing where that object will be located in the near future is of great importance in the field of motion analysis for applications such as security monitoring systems, $\mathrm{Au}-$ tonomous Driver Assistance Systems (ADAS), risk analysis and assistive technologies such as navigation for blind people to avoid collision.

Among motion prediction research, one specific task is path prediction, where the past positions (tracks) of objects are used to predict their future path. Several approaches have been developed (Madhavan et al., 2006; Schneider and Gavrila, 2013; Okamoto et al., 2017). Among recent approaches, LSTM architectures have been applied to this challenge due to their capability of getting information from sequences and then predicting using that previous information.

The main focus of this work is to show a technique that allows for forecasting a set of paths, along

\footnotetext{
a (iD) https://orcid.org/0000-0001-9774-3879

b (iD) https://orcid.org/0000-0003-3281-3471

c (iD) https://orcid.org/0000-0002-4033-9135
}

with their related probability, instead of a single one as in traditional approaches. We evaluate its performance on traffic scenarios from the KITTI (Geiger et al., 2013) and CityFlow (Tang et al., 2019) datasets to predict the future position of objects, such as pedestrians, vehicles and cyclists, for four prediction horizons (P.H.) on two selected datasets. In addition to using the most common position data (image coordinates in pixels), we also use a birds-eye view (metres) $(\mathrm{BEV})$ on the KITTI dataset since it is a more realistic measurement of the real world.

For the remainder of this paper, Section II presents relevant related works in this field, emphasising works using Long-Short Term Memory architectures (LSTMs) and Mixture Density Networks (MDNs). Section III describes the problem; Section IV presents our approach; Section V and VI present the experimental setup and results respectively. Finally in Section VII conclusions are given.

\section{RELATED WORKS}

A variety of techniques for path prediction have been developed, from the well known Kalman Filter (KF) (Kalman, 1960; Madhavan et al., 2006; Schnei- 
der and Gavrila, 2013; Jin et al., 2018), some probabilistic approaches (Keller and Gavrila, 2014), approaches based on prototype trajectories (Vasquez and Fraichard, 2004; Morris and Trivedi, 2008; Yoo et al., 2016; Bian et al., 2018) or based on manoeuvre intention (Madhavan et al., 2006; Keller and Gavrila, 2014) to Recurrent Neural Networks (RNNs) and its variants that have shown good performance on sequential data.

LSTM architectures are currently used in areas such as translation, time series prediction and trajectory prediction. LSTMs are capable of getting information from sequences and then predicting using that previous information.

One interesting work is shown in (Alahi et al., 2016), where they address the problem of predicting the trajectory of pedestrians in crowded spaces using static cameras. This approach, called Social LSTM, uses one LSTM for each of the pedestrians in the scene. "Social" refers to the use of the trajectory of other pedestrians that is taken into account to predict the trajectory of a single one. They use a separate LSTM for each trajectory and then connect each LSTM to other through a Social pooling layer. Similar work is presented in (Altché and de La Fortelle, 2017) where they use LSTMs to predict the trajectory of vehicles in highways from a fixed top-view.

In (Bartoli et al., 2018) multiple cameras were used to predict the trajectory of people in crowded scenes and (Kim et al., 2017) predict the trajectory of vehicles in an occupancy grid from the point of view of an ego-vehicle. A more closely related work to this paper is presented in (Bhattacharyya et al., 2017), here they predict the future path of pedestrians using RNNs as encoder-decoders and also include the prediction of the odometry of the ego-vehicle.

\subsection{Mixture Density Networks}

This type of network was introduced by (Bishop, 1994), Mixture Density Networks (MDNs) consist of a feed-forward neural network whose outputs determine the parameters in a mixture density model. The mixture model then represents the conditional probability density function of the target variables, conditioned in the input vector to the neural network.

Since then, MDNs have been applied on different works such as modeling of handwriting (Graves, 2013) or (Ha and Eck, 2017) for sketch drawing generation. At this point MDNs were not combined with standard Neural Networks, such as multi-layer perception, but with more complex architectures such as RNNs, more specifically with LSTMs. An interesting work is shown in (Ellefsen et al., 2019) where they generate images based on a sequence of past observed images using LSTMs and MDNs and also make a study on the role of the different mixture components.

A highly related work is presented in (Zyner et al., 2019), here the authors predict the intention of the driver (left, straight, right, u-turn) at 5 determined intersections in a static birds-eye view by predicting the future trajectories. In the intersection the possible trajectories of a vehicle are constrained to the five scenes and they apply clustering to the set of trajectories on the dataset to filter the predictions.

In this work, different to (Bartoli et al., 2018), path prediction is performed using cameras mounted on a moving vehicle and from surveillance. Instead of using one LSTM per object like in (Alahi et al., 2016), we use a common model for all objects of the same class. Also the prediction of the future path is made by two stacked vanilla LSTMs with a final MDN layer in a single-shot manner instead of using encoder-decoders or recursive multi-step forecasting. Different to (Zyner et al., 2019), we evaluate on three different object classes available in KITTI and one class in CityFlow dataset where more unconstrained scenarios than only intersections can be found. We also report the results from both, the image (pixels) and a birds-eye point of view (metres) using available $3 \mathrm{D}$ information.

\section{PROBLEM DEFINITION}

Path prediction research using RNNs has shown good performance. However, most of the approaches have the limitation of only predicting a single path per tracklet. Path prediction is not a deterministic task and requires predicting with a level of uncertainty. In addition, generating a set of paths instead of a single one is a more realistic manner of predicting the possible position of objects. Some works only focus on specific scenarios such as intersections, crossing roads and highways from a top view where the movements of the objects are limited by the shape of the scenarios. Nevertheless, real-life traffic scenarios are more diverse and consequently the movements of the objects in that environment are also diverse.

\subsection{Data Definition}

A path $P$ is a set of tracks, $t r$, that contains information such as $\operatorname{tr}(x, y)$ position (coordinates) of an object that travels a given space, $P=\left\{t r_{t 1}, t r_{t 2}, \ldots, t r_{\text {tlength }}\right\}$. Each $t r$ is a measure given for a sensor in intervals of time and in an ordered manner, $\operatorname{tr}(x, y$, time $)$. This means that a path is a sequence of measurements of 


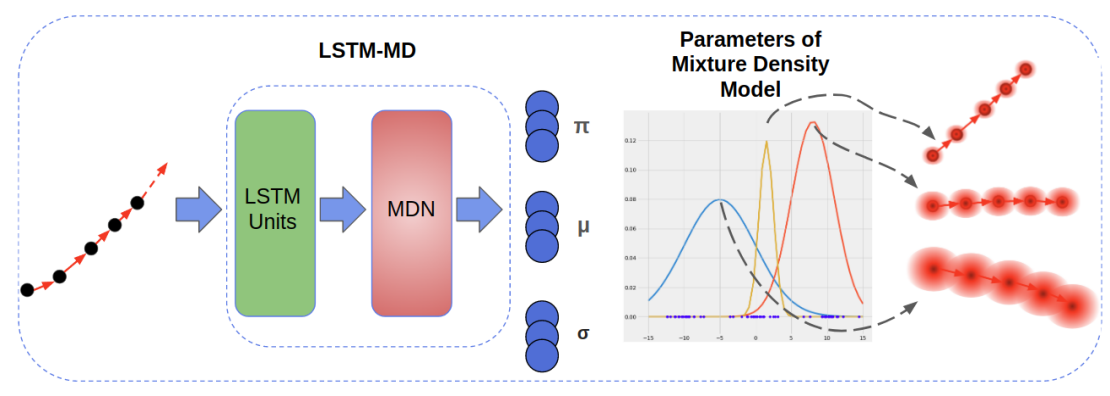

Figure 1: General Proposed Approach.

the same variable collected over time, where the order matters, resulting in a time series. Because of this, a path can be seen as a multivariate time series that has two time-dependent variables. Each variable depends on its past values, and this dependency is used for forecasting future values. So the task of path prediction can be seen as forecasting a multivariate multistep time series.

In this work we apply a sliding window over one track per time then these smaller segments are split into two vectors of equal size. The first vector is the observed tracklets $\operatorname{Tr}^{O}=\left[t r_{t 1}^{O}, t r_{t 2}^{O}, \ldots, t r_{\text {tobs }}^{O}\right]$ and the second vector is its respective ground truth tracklet $\operatorname{Tr}^{G}=\left[t r_{t 1}^{G}, t r_{t 2}^{G}, \ldots, t r_{t p r e d}^{G}\right]$. The predicted vector of each $\operatorname{Tr}^{O}$ is called $\operatorname{Tr}^{P}=\left[t r_{t 1}^{P}, t r_{t 2}^{P}, \ldots, t r_{\text {tpred }}^{P}\right]$. We aim to predict $\operatorname{Tr}^{P}$ based on the observed tracks $\operatorname{Tr}^{O}$ but instead of only predicting one $\operatorname{Tr}^{P}$ we want to predict a set, $\operatorname{Tr}^{P S}$, of $m \operatorname{Tr}^{P}$ per each $\operatorname{Tr}^{O}$ with its respective probability such that $\operatorname{Tr}^{P S}=\left[\operatorname{Tr}_{1}^{P}, \operatorname{Tr}_{2}^{P}, \ldots, \operatorname{Tr}_{m}^{P}\right]$ and $\operatorname{Tr}_{x}^{P}=\left[\operatorname{Tr}^{P}\right.$, Probability $]$ :

\section{APPROACH}

LSTMs have shown good performance when dealing with time series, so in this approach an LSTM architecture is used. LSTMs can be used in different manners, one is Multiple Output Strategy (MOS). MOS develops one model to predict an entire sequence in a one-shot manner, this output a vector directly that can be interpreted as a multi-step forecast. However, at this stage the problem of only being able to predict a single path per observed trajectory still remains. To overcome this limitation we use the well known properties of Mixture Density Models (MDMs) and inspired by (Bishop, 1994), we propose to use LSTMs with MDMs as a MDN layer, as shown in Figure 1.

\subsection{Model Architecture}

The core of the model are two stacked LSTMs with a final MDN layer. The number of inputs and outputs depends on the length of the observed tracklet, $\operatorname{Tr}^{O}$, and the number of steps to be predicted ahead, $\operatorname{Tr}^{P}$. The Keras API ${ }^{1}$ and the Keras MDN Layer library ${ }^{2}$ were used to obtain the implementation of the LSTM architecture and the MDN Layer respectively.

\subsection{Multiple Trajectory Extraction}

For this phase, the output of the model was processed as follow:

1. Extracting mean, standard deviation and mixing proportions from output. The model outputs a single array per $\operatorname{Tr}^{O}$ where the first NMixes* Out putLength columns are the means, the second NMixes $*$ Out putLength columns are the standard deviations and the last NMixes are the mixing proportions.

2. Each mean is considered as a possible path and its mixing proportion is the probability of each path.

\section{EXPERIMENTAL SETUP}

\subsection{Datasets}

Two datasets were chosen:

- KITTI (Geiger et al., 2013): provides information recorded from a camera mounted on a vehicle and is one of the most popular datasets for use in mobile robotics and autonomous driving. It also provides 21 sequences with the tracking labels of the objects in image coordinates and 3D information, as visualised in Fig. 2. The resolution of the videos is $1242 \times 375 \mathrm{p}$ and are recorded at $10 \mathrm{FPS}$.

- CityFlow (Tang et al., 2019): provides information on objects from surveillance cameras in image coordinate format. The minimum video resolution is $1920 \times 1080 \mathrm{p}$ and the majority of the

\footnotetext{
${ }^{1}$ https://keras.io/

${ }^{2}$ https://pypi.org/project/keras-mdn-layer/
} 
videos have a frame rate of 10 FPS. The three scenarios from training were used, since only these scenarios contain the tracking labels of the objects.

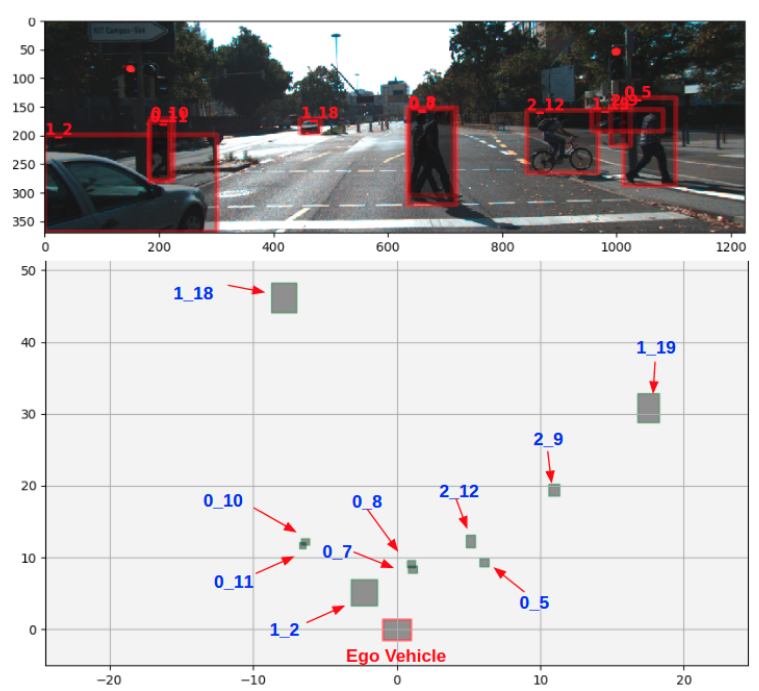

Figure 2: Image coordinates (top) and birds-eye view (bottom).

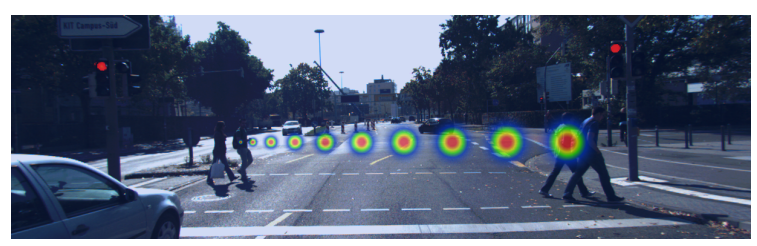

Figure 3: Heat maps of 10-100 pixels (left to right) illustrating pixel differences in the real world.

\subsection{Data Pre-processing}

The data was pre-processed as follows:

1. Convert the dataset to a simpler format with each track described as follows: [Frame-Num, Object-Type, Object-Id, X1, Y1, X2, Y2, Location-X, Location-Y, Location-Z, DimensionH, Dimension-W, Dimension-L] for KITTI and [Frame-Num, Object-Type, Object-Id, X1, Y1, X2, Y2] for CityFlow.

2. Extract trajectories of each object per sequence.

3. Create tracklets (sub-trajectories) of a certain length. For each object trajectory, tracklets of size $10,20,30$ and 40 tracks were extracted.

4. To extract the bottom center of the objects.

5. To translate the tracklets to relative position. This process consists of setting the first $(x, y)$ position of each tracklet to $(0,0)$ and all the following tracks are adjusted relative to this point.

6. Normalize all tracklets to $[0,1]$.

The table 1 summarizes the number of tracklets extracted in each dataset. $70 \%$ was used for training and the rest for testing:

Table 1: Size of the data for all four P.H. and objects.

\begin{tabular}{|l|c|c|c|c|}
\hline \multicolumn{5}{|c|}{ Number of Tracklets } \\
\hline & \multicolumn{3}{|c|}{ KITTI } & CityFlow \\
\hline P.H. & Pedestrian & Vehicle & Cyclist & Vehicle \\
\hline \pm 5 & 9992 & 26112 & 1605 & 37480 \\
\hline \pm 10 & 8551 & 20454 & 1285 & 31164 \\
\hline \pm 15 & 7330 & 16031 & 1028 & 25936 \\
\hline \pm 20 & 6312 & 13027 & 802 & 21923 \\
\hline
\end{tabular}

\subsection{Evaluation metrics}

The following metrics were used to evaluate the accuracy of the trajectory prediction (Alahi et al., 2016):

- Average Displacement Error (ADE): is the mean square error (MSE) between all estimated points of every trajectory and the true points:

$$
A D E=\frac{\sum_{i=1}^{n} \sum_{t=1}^{t \text { pred }}\left[\left(\hat{x}_{i}^{t}-x_{i}^{t}\right)^{2}+\left(\hat{y}_{i}^{t}-y_{i}^{t}\right)^{2}\right]}{n(\text { tpred })}
$$

- Final Displacement Error (FDE): is the distance between the predicted final destination and the true final destination at the tpred time:

$$
F D E=\frac{\sum_{i=1}^{n}\left[\left(\hat{x}_{i}^{\text {tpred }}-x_{i}^{\text {tpred }}\right)^{2}+\left(\hat{y}_{i}^{t \text { pred }}-y_{i}^{\text {tpred }}\right)^{2}\right]}{n}
$$

where $\left(\hat{x}_{i}^{t}, \hat{y}_{i}^{t}\right)$ are the predicted positions of the tracklet $i$ at time $t,\left(x_{i}^{t}, y_{i}^{t}\right)$ are the actual position (ground truth) of the tracklet $i$ at time $t$, and $n$ is the number of tracklets in the testing set.

\subsection{Comparative study}

We compare our approach with two baselines methodologies to establish that our approach does not lose accuracy when predicting a set of paths.

- The Kalman Filter (KF): the KF was used with the Constant Velocity (CV) model. This model has shown good performance when dealing with linear movements.

- Vanilla LSTM (VLSTM): this consists of a one layer LSTM with 128 neurons. This model was also used in a single-shot manner. 
For our proposed model (LSTM with MDM), we performed two experiments for image coordinates. Instead of only using two features, $(x, y)$ position of objects (LMDN2), we included three additional features: height, object area and object area with respect to the image (LMDN5). This produced a feature vector of $(x, y, h$, object A, objectAImg).

\section{RESULTS}

The performance was calculated on four different prediction horizons (P.H.) and for three different objects - pedestrians, cyclists and vehicles (Cars, Vans or Trucks). The results are also provided in image coordinates (pixels) and in birds-eye view (metres). Due to the size of the images, the results show large numerical values in the case of image coordinates. Fig. 3 illustrates the approximate real world implication of variations in pixels as applied to the KITTI dataset. Finally, the approach was also evaluated on the generation of different numbers of paths, two to five. The results (Tables 2, $3 \& 4$ ) show the accuracy of the mixture component with the highest probability and compared with the baseline methods. Examples of predicting different numbers of paths are shown in the visualizations (Fig. 4 \& 5).

\subsection{KITTI}

Table 2 shows the performance of the approach for image coordinates. For all methods, the error increases directly with the predicted horizon; the larger the P.H. the larger the error. Table 2 shows that our approach LMDN2 achieves better accuracy than the two baseline methods. It can also be observed that the approach LMDN5 showed improvement for short P.H. in ADE and in several cases for FDE, mostly for the objects pedestrian and vehicle.

Table 3 presents the results calculated using birdseye view (BEV). For most of the cases our approach, LMDN2, achieves better accuracy than the two baseline methods. An exception is a significant error increase in the case of the vehicle class for the P.H. of \pm 10 for both ADE and FDE. Figure 4 shows the resulting set of paths predicted when configuring the model to have five mixtures. The first 1,000 samples were printed. The first image (GT) displays the ground truth paths and the following images from MDN 1 to MDN 5 present the paths predicted by each mixture component. The mixtures were ordered according to descending probability of their components - MDN 1 (high probability) to MDN 5 (low probability). The predicted paths diverge from the ground truth when the probability of the the components used for predicting go from high to low.

\subsection{CityFlow}

Table 4 presents the performance of the methods on the CityFlow dataset. For all approaches, similar behaviour to that in KITTI can be observed - the error increases directly with the P.H. Table 4, shows that our approach LMDN2 achieves better accuracy than the two baseline methods. The approach LMDN5 (using 5 features) showed significant improvement over the method LMDN2 for the P.H. of $\pm 5, \pm 10$, and \pm 15 for both $\mathrm{ADE}$ and FDE.

To predict a set of paths, from 2 to 5 , similar behaviour to that in KITTI is observed. The predicted paths diverge from the ground truth when the probability of the components used for predicting goes from high to low. Figure 5 depicts one example of predicting from 2 to 5 sets of paths for a P.H. of \pm 5 . When the predicted paths are near the GT, the probability of such a path is high, in contrast, when the predicted paths are far from the GT, their probability is low. In some cases, as in figure 5 at $\mathrm{C}$ and D left, the paths are not displayed because the probability of that path is too low. Scatter plots are an alternative visualisation of the predicted paths but doesn't include their probabilities.

\subsection{Discussion}

The comparative study shows that LSTMs do not decrease their performance when combined with the MDN. Regarding the method LMDN5, the experiments showed that the extra features lead to better results overall. This was evidenced in KITTI for short prediction horizons for the object pedestrian and vehicle and for CityFlow for the object vehicle for P.H. of less than \pm 15 . The relationship between the accuracy of the predicted set of paths and the probability of each component in the MDN model can be seen in Figure 4 and Figure 5. The predicted paths are more similar to the ground truth when the component that is predicting has high probability. However, those paths that are being predicted for the components with low probability are increasingly different to ground truth. This conclusion is desirable when predicting paths, since we want to predict possible paths that are closer to the more probable one.

The approach performs better when predicting in birds-eye view than when predicting in image coordinates. However, 3D information is not always available. The reason for this could be that using pixels is not the best way of representing the position 
Table 2: Path prediction accuracy on KITTI. Image coordinates.

\begin{tabular}{|c|c|c|c|c|c|c|c|c|c|c|c|c|}
\hline \multicolumn{13}{|c|}{ KITTI. Image Coordinate (Pixels) } \\
\hline Method & \multirow{2}{*}{\multicolumn{3}{|c|}{$\frac{\text { LMDN5 }}{\text { ADE }}$}} & \multirow{2}{*}{\multicolumn{3}{|c|}{$\frac{\text { LMDN2 }}{\text { ADE }}$}} & \multirow{2}{*}{\multicolumn{3}{|c|}{$\begin{array}{c}\text { VLSTM } \\
\text { ADE }\end{array}$}} & \multicolumn{3}{|c|}{ KF } \\
\hline & \multicolumn{2}{|c|}{ ADE } & & & & & & & & \multicolumn{3}{|c|}{ ADE } \\
\hline P. H. & Ped. & Veh. & Cyc. & Ped. & Veh. & Cyc. & Ped. & Veh. & Cyc. & Ped. & Veh. & Cyc. \\
\hline$\pm \mathbf{5}$ & 79 & 65 & & 106 & 84 & 10 & 76 & 98 & 94 & 111 & 225 & 143 \\
\hline$\pm \mathbf{1 0}$ & 86 & 272 & 32 & 362 & 267 & 26 & 244 & 353 & 160 & 260 & 585 & 287 \\
\hline \pm 15 & 466 & 591 & 1596 & 660 & 614 & 467 & 802 & 668 & 1163 & 549 & 1019 & 807 \\
\hline$\pm \mathbf{2 0}$ & 07 & 1035 & 15673 & 85 & 723 & 2854 & 755 & 1065 & 6856 & 970 & 1554 & 1944 \\
\hline & \multicolumn{3}{|c|}{ FDE } & \multicolumn{3}{|c|}{ FDE } & \multicolumn{3}{|c|}{ FDE } & \multicolumn{3}{|c|}{ FDE } \\
\hline P. H. & Ped. & Veh. & Cyc. & Pe. & Veh. & Сус & Ped. & Veh. & Cyc. & Ped. & Veh. & Сус. \\
\hline \pm 5 & 168 & 161 & 502 & 237 & 220 & 277 & 169 & 252 & 271 & 214 & 501 & 385 \\
\hline$\pm \mathbf{1 0}$ & 721 & 896 & 1124 & 1098 & 924 & 946 & 727 & 1163 & 538 & 778 & 1914 & 1022 \\
\hline \pm 15 & 1517 & 2265 & 3742 & 2231 & 2230 & 1917 & 2483 & 2412 & 4452 & 1907 & 3695 & 3408 \\
\hline$\pm \mathbf{2 0}$ & 5061 & 3678 & 38492 & 4100 & 2828 & 12346 & 6251 & 4040 & 25743 & 3567 & 5808 & 8539 \\
\hline
\end{tabular}

Table 3: Path prediction accuracy on KITTI. BEV.

\begin{tabular}{|l|r|r|r|r|r|r|r|r|r|}
\hline \multicolumn{9}{|c|}{ KITTI. Bird-Eye View (Meters) } \\
\hline Method & \multicolumn{3}{|c|}{ LMDN2 } & \multicolumn{3}{c|}{ VLSTM } & \multicolumn{3}{c|}{ KF } \\
\hline \multicolumn{9}{|c|}{ ADE } & \multicolumn{3}{|c|}{ ADE } & \multicolumn{3}{c|}{ ADE } \\
\hline P. H. & Ped. & Veh. & Cyc. & Ped. & Veh. & Cyc. & Ped. & Veh. & Cyc. \\
\hline$\pm \mathbf{5}$ & 0.01 & 0.06 & 0.02 & 0.01 & 0.06 & 0.02 & 0.06 & 0.47 & 0.24 \\
\hline$\pm \mathbf{1 0}$ & 0.04 & 0.55 & 0.11 & 0.05 & 0.25 & 0.10 & 0.10 & 0.75 & 0.36 \\
\hline$\pm \mathbf{1 5}$ & 0.10 & 0.73 & 0.285 & 0.12 & 0.87 & 0.41 & 0.21 & 1.12 & 0.54 \\
\hline$\pm \mathbf{2 0}$ & 0.31 & 1.31 & 0.805 & 0.22 & 1.68 & 1.01 & 0.41 & 1.79 & 0.90 \\
\hline \multicolumn{8}{|c|}{ FDE } & \multicolumn{3}{|c|}{ FDE } & \multicolumn{5}{c|}{ FDE } \\
\hline P. H. & Ped. & Veh. & Cyclist & Ped. & Veh. & Cyc. & Ped. & Veh. & Cyc. \\
\hline$\pm \mathbf{5}$ & 0.02 & 0.13 & 0.03 & 0.02 & 0.15 & 0.04 & 0.08 & 0.58 & 0.27 \\
\hline$\pm \mathbf{1 0}$ & 0.13 & 1.24 & 0.29 & 0.14 & 0.72 & 0.27 & 0.25 & 1.67 & 0.64 \\
\hline$\pm \mathbf{1 5}$ & 0.32 & 2.16 & 1.08 & 0.38 & 2.69 & 1.29 & 0.69 & 3.28 & 1.42 \\
\hline$\pm \mathbf{2 0}$ & 1.13 & 4.21 & 2.21 & 0.77 & 5.48 & 3.06 & 1.49 & 6.04 & 2.95 \\
\hline
\end{tabular}

Table 4: Path prediction accuracy on CityFlow. Image coordinates.

\begin{tabular}{|l|c|c|c|c|}
\hline \multicolumn{5}{|c|}{ CityFlow. Image Coordinate (Pixels). } \\
\hline Method & LMDN5 & LMDN2 & VLSTM & \multicolumn{1}{c|}{ KF } \\
\hline P.H. & ADE & \multicolumn{1}{c|}{ ADE } & \multicolumn{1}{c|}{ ADE } & ADE \\
\hline$\pm \mathbf{5}$ & 486 & 582 & 634 & 1369 \\
\hline$\pm \mathbf{1 0}$ & 665 & 905 & 893 & 1948 \\
\hline$\pm \mathbf{1 5}$ & 891 & 1094 & 1134 & 1846 \\
\hline$\pm \mathbf{2 0}$ & 1613 & 1371 & 1587 & 2202 \\
\hline $\mathbf{P . H .}$ & FDE & FDE & FDE & FDE \\
\hline$\pm \mathbf{5}$ & 878 & 1125 & 1209 & 2492 \\
\hline$\pm \mathbf{1 0}$ & 1792 & 2659 & 2525 & 5167 \\
\hline$\pm \mathbf{1 5}$ & 3084 & 3817 & 4135 & 6585 \\
\hline$\pm \mathbf{2 0}$ & 5749 & 5197 & 6121 & 8382 \\
\hline
\end{tabular}

of the object in an image since is too sensitive to small movements of the camera and also of the objects. Something to consider here is that when predicting in image coordinates, further normalisation is needed to counter the size of the images of the dataset. Results in pixels were provided here to be consistent with other published results. As shown in our experiments, the errors in pixels are large but that does not mean that the predictions are far from the ground truth paths.

Finally, the processing inference time per tracklet for our approach was $0.044 \mathrm{~ms} / \mathrm{tr}, 0.055 \mathrm{~ms} / \mathrm{tr}$, $0.084 \mathrm{~ms} / \mathrm{tr}, 0.102 \mathrm{~ms} / \mathrm{tr}$ for P.H. of \pm 5 to \pm 20 respectively. This was measured using a PC with the following features: GPU GeForce GTX 980, CPU Intel $\AA$ Core $^{\mathrm{TM}}$ i5-4690K CPU @ 3.50GHz x 4, RAM 24GB.

\section{CONCLUSIONS}

We present an approach for predicting multiple paths with associated uncertainty for forecasting possible near future position of objects commonly present in traffic scenes. The objective of this work was to explore the performance of the combination of LSTM and MDN architectures and analyze the parameters output by these models for predicting a set of paths. The evaluation was made for three object classes, four P.H and two different points of view. 

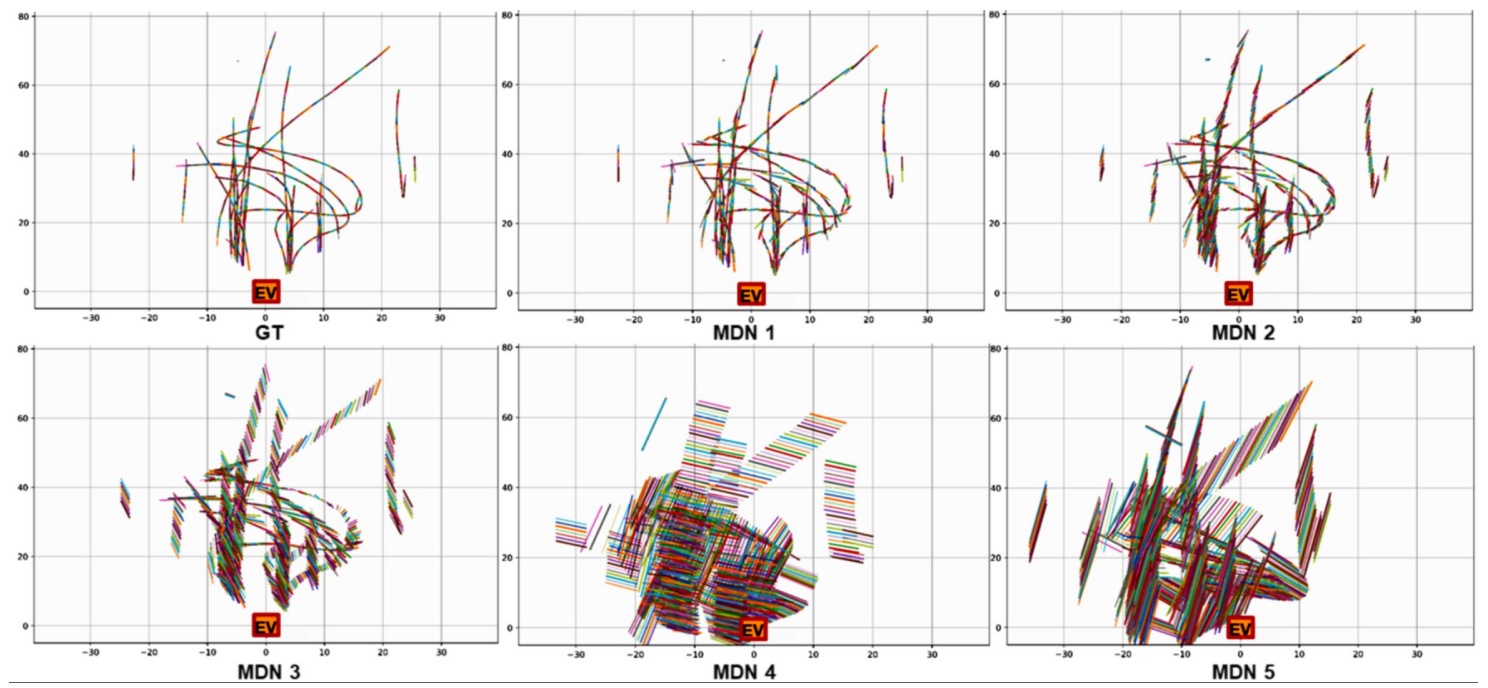

Figure 4: Set of paths predicted using five mixtures. Dataset: KITTI. P.H.: \pm 5. Point of view: BEV.
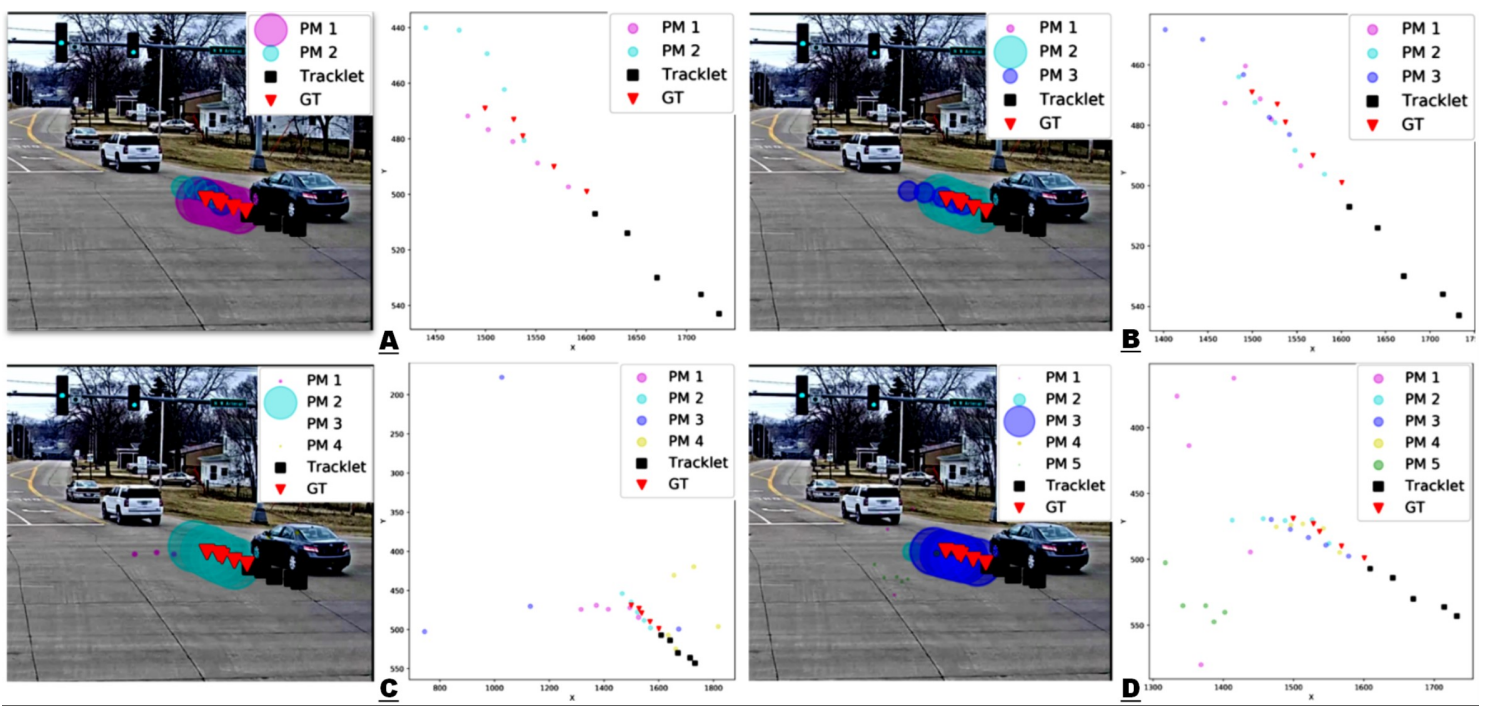

Figure 5: Predicting two (A), three (B), four (C) and five (D) set of paths. Left: shows the predicted set of paths with their respective probability (the larger the circle, the larger the probability of that path). Right: presents a close-up of the set of paths without their probabilities. Dataset: CityFlow. P.H.: \pm 5. Point of view: Image Coordinate.

The experiments shown that the combination of LSTMs and MDNs does not reduce overall performance and in some cases the accuracy was improved. It can also be seen that including more features to the tracklets leads to better accuracy. The results have shown that the approach achieves good performance of up to an ADE of $0.01 \mathrm{~m}$ for pedestrians, $0.06 \mathrm{~m}$ for vehicles and $0.02 \mathrm{~m}$ for cyclists and up to an FDE of $0.02 \mathrm{~m}, 0.13 \mathrm{~m}, 0.03 \mathrm{~m}$ for the same objects using BEV and P.H. of \pm 5 . The results also show that the performance is affected by the P.H. where longer horizons result in a larger displacement error. The P.H. where the approach is more reliable is for \pm 5 and \pm 10 for image coordinate and up to \pm 15 for birds-eye view.
The FPS in both datasets is 10 therefore we are predicting from $( \pm 0.5 \mathrm{~s})$ to $( \pm 2 \mathrm{~s})$ seconds ahead.

The approach was also evaluated for predicting multiple numbers of paths per input tracklet. It was observed that when predicting two to three paths per input the approach works well as the predicted paths are still related to the ground truth (GT). However, in some cases, when predicting four and five paths, some of the predicted paths begin to deviate further from the GT. This cannot be seen as a disadvantage since each path has a probability, so by looking at the probability of each path, those paths with very low probability can be discarded.

This work uses positional and observed paths 
only, the next step will be to combine external data to constrain the path prediction based on real world knowledge. Semantic segmentation in traffic scenes is relatively reliable, so predicted path may be constrained by applying a semantic segmentation map of the scenes and removing those paths that are not adjacent to regions classified as road and sidewalk. Specifically for the case of cameras mounted on a vehicle, the next work is to include the ego-motion. Finally, to tackle the problem of representing the position of objects on an image coordinate by pixels and its sensitivity, it would be interesting to see the image as a grid, and represent the position of the object according to this grid.

\section{ACKNOWLEDGEMENTS}

This work has received funding from EU H2020 Project VI-DAS under grant number 690772 and Insight Centre for Data Analytics funded by SFI, grant number SFI/12/RC/2289. The GPU GeForce GTX 980 used for this research was donated by the NVIDIA Corporation.

\section{REFERENCES}

Alahi, A., Goel, K., Ramanathan, V., Robicquet, A., FeiFei, L., and Savarese, S. (2016). Social LSTM: Human trajectory prediction in crowded spaces. In Proceedings of the IEEE conference on computer vision and pattern recognition, pages 961-971.

Altché, F. and de La Fortelle, A. (2017). An LSTM network for highway trajectory prediction. In 2017 IEEE 20th International Conference on Intelligent Transportation Systems (ITSC), pages 353-359. IEEE.

Bartoli, F., Lisanti, G., Ballan, L., and Del Bimbo, A. (2018). Context-aware trajectory prediction. In 2018 24th International Conference on Pattern Recognition (ICPR), pages 1941-1946. IEEE.

Bhattacharyya, A., Fritz, M., and Schiele, B. (2017). Long-term on-board prediction of pedestrians in traffic scenes. In 1st Conference on Robot Learning.

Bian, J., Tian, D., Tang, Y., and Tao, D. (2018). A survey on trajectory clustering analysis. arXiv preprint arXiv:1802.06971.

Bishop, C. M. (1994). Mixture density networks.

Ellefsen, K. O., Martin, C. P., and Torresen, J. (2019). How do mixture density rnns predict the future? arXiv preprint arXiv:1901.07859.

Geiger, A., Lenz, P., Stiller, C., and Urtasun, R. (2013). Vision meets robotics: The KITTI dataset. The International Journal of Robotics Research, 32(11):12311237.
Graves, A. (2013). Generating sequences with recurrent neural networks. arXiv preprint arXiv:1308.0850.

Ha, D. and Eck, D. (2017). A neural representation of sketch drawings. arXiv preprint arXiv:1704.03477.

Jin, X.-B., Su, T.-L., Kong, J.-L., Bai, Y.-T., Miao, B.-B., and Dou, C. (2018). State-of-the-Art mobile intelligence: Enabling robots to move like humans by estimating mobility with artificial intelligence. Applied Sciences, 8(3):379.

Kalman, R. E. (1960). A new approach to linear filtering and prediction problems. Journal of basic Engineering, 82(1):35-45.

Keller, C. G. and Gavrila, D. M. (2014). Will the pedestrian cross? a study on pedestrian path prediction. IEEE Transactions on Intelligent Transportation Systems, 15(2):494-506.

Kim, B., Kang, C. M., Kim, J., Lee, S. H., Chung, C. C., and Choi, J. W. (2017). Probabilistic vehicle trajectory prediction over occupancy grid map via recurrent neural network. In 2017 IEEE 20th International Conference on Intelligent Transportation Systems (ITSC), pages 399-404. IEEE.

Madhavan, R., Kootbally, Z., and Schlenoff, C. (2006). Prediction in dynamic environments for autonomous onroad driving. In 2006 9th International Conference on Control, Automation, Robotics and Vision, pages 1-6. IEEE.

Morris, B. T. and Trivedi, M. M. (2008). Learning and classification of trajectories in dynamic scenes: A general framework for live video analysis. In Advanced Video and Signal Based Surveillance, 2008. AVSS'08. IEEE Fifth International Conference on, pages 154161. IEEE.

Okamoto, K., Berntorp, K., and Di Cairano, S. (2017). Similarity-based vehicle-motion prediction. In 2017 American Control Conference (ACC), pages 303-308. IEEE.

Schneider, N. and Gavrila, D. M. (2013). Pedestrian path prediction with recursive bayesian filters: A comparative study. In German Conference on Pattern Recognition, pages 174-183. Springer.

Tang, Z., Naphade, M., Liu, M.-Y., Yang, X., Birchfield, S., Wang, S., Kumar, R., Anastasiu, D., and Hwang, J.-N. (2019). CityFlow: A city-scale benchmark for multi-target multi-camera vehicle tracking and reidentification. In The IEEE Conference on Computer Vision and Pattern Recognition (CVPR).

Vasquez, D. and Fraichard, T. (2004). Motion prediction for moving objects: a statistical approach. In Proceedings of the IEEE International Conference on Robotics and Automation (ICRA), volume 4, pages 3931-3936.

Yoo, Y., Yun, K., Yun, S., Hong, J., Jeong, H., and Young Choi, J. (2016). Visual path prediction in complex scenes with crowded moving objects. In Proceedings of the IEEE Conference on Computer Vision and Pattern Recognition, pages 2668-2677.

Zyner, A., Worrall, S., and Nebot, E. (2019). Naturalistic driver intention and path prediction using recurrent neural networks. IEEE Transactions on Intelligent Transportation Systems. 